\title{
Percent Change From Baseline in Sum of Diameter
}

National Cancer Institute

\section{Source}

National Cancer Institute. Percent Change From Baseline in Sum of Diameter. NCI

Thesaurus. Code C112370.

The most recently recorded sum of diameters minus the baseline sum of diameters divided by the baseline sum of diameters, multiplied by 100 . 